5

6

7

8

9

\title{
Interfacial characteristics and microchannel emulsification of oleuropein-containing triglyceride oil-water systems
}

Safa Souilema ${ }^{\mathrm{a}, \mathrm{b}, \mathrm{c}, \mathrm{d}}$, Isao Kobayashib,c,*, Marcos A. Neves ${ }^{\mathrm{a}, \mathrm{b}, \mathrm{c}}$, Lobna Jlaiel ${ }^{\mathrm{d}}$, Hiroko Isoda ${ }^{\mathrm{a}, \mathrm{c}}$, Sami Sayadid ${ }^{\text {, Mitsutoshi Nakajima }}{ }^{\mathrm{a}, \mathrm{b}, \mathrm{c}, * *}$

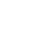

${ }^{a}$ Graduate School of Life and Environmental Sciences, University of Tsukuba, Tsukuba, 1-1-1

Tennoudai, Tsukuba, Ibaraki, 305-8572, Japan

${ }^{\mathrm{b}}$ Food Engineering Division, National Food Research Institute, NARO, Tsukuba, 2-1-12

Kannondai, Tsukuba, Ibaraki, 305-8642, Japan

${ }^{\mathrm{c}}$ Alliance for Research on North Africa, University of Tsukuba, Tsukuba, 1-1-1 Tennoudai,

Tsukuba, Ibaraki 305-8572, Japan

${ }^{\mathrm{d}}$ Center of Biotechnology of Sfax, B.P. 1177, Sfax 3018, Tunisia

* Corresponding author. Tel.: +81 29838 8025; fax: +81 298388122.

E-mail address: isaok@affrc.go.jp (I. Kobayashi)

** Corresponding author. Tel.: +81298534703; fax : +81298534703

E-mail address: nakajima.m.fu@u.tsukuba.ac.jp (M. Nakajima)

Keywords: Oleuropein, Olive leaf extract, Interfacial activity, Gibbs adsorption equation, Triglyceride oil-in-water emulsion, Microchannel emulsification 
This study investigated the adsorption characteristics of olive leaf water extract and its major phenolic compound, oleuropein, at the triglyceride oil-water interface. We also investigated the preparation characteristics of food-grade triglyceride oil-in-water $(\mathrm{O} / \mathrm{W})$ emulsions stabilized by oleuropein using microchannel (MC) emulsification. Refined soybean oil, extra virgin olive oil, refined olive oil, and medium-chain triacylglyceride (MCT) oil were used as triglyceride oils. Both olive leaf extract (OLE) and highly purified oleuropein had a pronounced ability to decrease the interfacial tension at the refined soybean oil-water interface. The packing of oleuropein molecules at the triglyceride oil-water interface was estimated on the basis of their surface excess concentration and area occupied per molecule, determined from the Gibbs adsorption equation. $\mathrm{MC}$ emulsification was performed using a silicon grooved MC array plate (model CMS6-2). The continuous aqueous phase contained $0.6 \mathrm{wt} \%$ of oleuropein. Monodisperse, oleuropein-stabilized $\mathrm{O} / \mathrm{W}$ emulsions with an average droplet diameter of $25 \mu \mathrm{m}$ and coefficient of variation $(\mathrm{CV})$ of $<5 \%$ were produced in all systems, except the MCT oil-containing system, even in the absence of a cross-flowing continuous phase. This successful MC emulsification was observed without droplet coalescence for $15 \mathrm{~h}$ of continuous operation. Our findings demonstrate that the use of oleuropein, which has an interfacial activity, is capable of producing monodisperse $\mathrm{O} / \mathrm{W}$ emulsions using $\mathrm{MC}$ emulsification and stabilizing the generated oil droplets when appropriate types of triglyceride oils are used. 


\section{Introduction}

43 Numerous food emulsion products (e.g., butter, mayonnaise, sauces, and creams) are

44 commercially available. Food emulsions are normally composed of many ingredients (e.g., 45 oils, emulsifiers, thickening agents, preservatives, antioxidants, colorants, and flavors) 46 (McClements., 2005). The functionality of a food emulsion depends on its microstructure and 47 is influenced by the droplet concentration, droplet size distribution, interfacial characteristics, and physical state of the droplets (Terjung, Loffler, Gibis, Hinrichs, \& Weiss, 2012). The emulsifier is an important emulsion ingredient because its adsorption at the oil-water interface can significantly lower interfacial tension and prevent droplet aggregation and coalescence. Therefore, it is crucial to choose the appropriate emulsifier to produce stable emulsions. Seemingly, understanding the functions of other ingredients and their possible effects on the physicochemical and organoleptic properties of emulsions is of great importance.

Antioxidants are usually used to control lipid oxidation in most food emulsions. In addition to their chemical properties, the effectiveness of antioxidants in emulsions can also depend on their surface properties and ability to accumulate at the interface, which is where oxidation commences (Yuji et al., 2007). Previous studies have quantitatively determined the number of antioxidant molecules that accumulate at the oil-water interface of emulsions stabilized by emulsifier molecules (Paiva-Martins, Gordon, \& Gameiro, 2003; Viljanen, Kylli, Hubbermann, Schwarz, \& Heinonen, 2005). The effect of the molecular characteristics of antioxidants (e.g., polarity, molecular weight, and surface activity) on their partitioning and effectiveness in food emulsions was explained by the "polar paradox" phenomenon (Chaiyasit, McClements, \& Decker, 2005; Laguerre et al., 2009; Richards, Chaiyasit, McClements, \& Decker, 2002). This phenomenon means that polar antioxidants are more effective in nonpolar lipids, while nonpolar antioxidants are more effective in oil-in-water 
$(\mathrm{O} / \mathrm{W})$ emulsions. The interfacial activity of antioxidants can be increased by their conjugation to fatty acid or fatty alcohol chains (Lucas et al., 2010; Medina, Lois, Alcántara, Lucas, \& Morales, 2009)

In addition to antioxidants, antimicrobials are incorporated in food emulsions to inhibit microbial growth, allowing for longer shelf lives. Antioxidants are reported to enhance the stability of O/W emulsions (Di Mattia, Sacchetti, Mastrocola, Sarker, \& Pittia, 2010; Kargar, Spyropoulos, \& Norton, 2011), whereas only a few studies have addressed the effect of antimicrobials on the physicochemical properties of emulsions (J. Han \& Washington, 2005; Yuan, Wan, Yin, \& Yang, 2013). Potent antimicrobials, paraben and benzoic acid, reduce the stability of $\mathrm{O} / \mathrm{W}$ emulsions; however, a mixture of $\beta$-conglycinin and chitosan increased the stability of a soy protein-stabilized emulsion (Goy, Britto, \& Assis, 2009; Sitohy, Mahgoub, \& Osman, 2012; Yuan et al., 2013).

Because of the increasing demand for natural preservatives in foods, olive leaves have received a great deal of attention as an abundant plant material containing various phenolic compounds (e.g., secoiridoids and flavonoids). Oleuropein, the main olive leaf phenolic compound (6.8 wt\% per unit dry weight), is considered to be a potent antimicrobial and antioxidant agent (Andreadou et al., 2006; Benavente-García, Castillo, Lorente, Ortuño, \& Del Rio, 2000; Bouaziz \& Sayadi, 2005). It also exhibits antidiabetic (Jemai, El Feki, \& Sayadi, 2009) and anticancer (Junkyu, Talorete, Yamada, \& Isoda, 2009) characteristics. For this reason, olive leaves (mainly oleuropein) have great potential to be used in the development of natural food and pharmaceutical products. Oleuropein (Fig. 1) is a monoterpenoid glycoside synthesized via a branching of mevalonic acid during the formation of oleosides in olive leaves (Omar 2010). It is assumed that the high antiviral and antimicrobial activities of oleuropein against both Gram-negative and Gram-positive bacteria 
are partially dependent on its surface activity at the bacterial cell membrane (Bisignano et al., 1999; Casas-Sanchez, Alsina, Herrlein, \& Mestres, 2007; Juven, Henis, \& Jacoby, 1972). The antioxidant activity of oleuropein within phospholipid liposomes was also partly associated with its location at the surface of their bilayer (Paiva-Martins et al., 2003). A recent study confirmed that the presence of oleuropein could significantly reduce the interfacial tension between refined olive oil and water (Mattia, Sacchetti, \& Pittia, 2011), confirming its high surface activity. Many studies have focused on quantitatively determining the quantity of antioxidants that accumulate at the oil-water interface of emulsions in the presence of emulsifiers (Paiva-Martins et al., 2003; Viljanen et al., 2005). However, to the best of our knowledge, the adsorption characteristics of oleuropein at a pure triglyceride oil-water interface have not yet been investigated.

Food emulsions are usually produced using conventional emulsification devices (e.g., highspeed blenders, colloid mills, and high-pressure homogenizers) (McClements., 2005). These devices are advantageous for mass-producing emulsions over a wide range of droplet sizes (typically from 0.1 to $100 \mu \mathrm{m}$ ). However, the energy input during emulsification is mostly converted to heat (i.e., temperature elevation) (Gijsbertsen-Abrahamse, van der Padt, \& Boom, 2004), degrading heat-sensitive bioactives such as polyphenols and vitamins (Solans, Izquierdo, Nolla, Azemar, \& Garcia-Celma, 2005). Moreover, using conventional emulsification devices results in polydisperse emulsions with empirically controlled droplet sizes. Our research group introduced microchannel (MC) emulsification using unique MC arrays, which each consist of parallel MCs and terraces outside the arrays (Kawakatsu, Kikuchi, \& Nakajima, 1997). The major advantages of MC emulsification include production of monodisperse emulsions with precisely controlled droplet sizes, low energy input (typically $10^{3}-10^{4} \mathrm{~J} / \mathrm{m}^{3}$ ) that prevents temperature elevation, and remarkably mild droplet generation in 
the absence of external forces (Sugiura, Nakajima, Kumazawa, Iwamoto, \& Seki, 2002; Vladisavljević, Kobayashi, \& Nakajima, 2012). Current MC emulsification devices enable production of monodisperse emulsions with average droplet diameters of 1-550 $\mu \mathrm{m}$ and coefficients of variation (CVs) of $<5 \%$ (Isao Kobayashi et al., 2008; I. Kobayashi et al., 2012). Various up-to-date emulsifiers and stabilizers have been used for MC emulsification research. However, polyphenols have not yet been considered as surface-active compounds.

The first objective of this work was to systematically characterize the adsorption of olive leaf water extract and its major phenolic compound, oleuropein, at triglycerol oil-water interfaces on the basis of the Gibbs adsorption theory. The second objective was to evaluate the ability of oleuropein to stabilize the triglycerol oil-water interface using MC emulsification. The influence of operating conditions on the generation of oil droplets via $\mathrm{MC}$ arrays was also investigated.

\section{Materials and methods}

\subsection{Materials}

Refined soybean oil and refined olive oil were purchased from Wako Pure Chemical Industries, Ltd. (Osaka, Japan). Medium-chain triglyceride (MCT; Sunfat MCT-7) oil was obtained from Taiyo Kagaku Co. Ltd. (Mie, Japan). Extra virgin olive oil was purchased at a local market in Tunisia. Oleuropein (94\% purity), tyrosol, luteolin, and luteolin glycoside were purchased from Extrasynthese Co. (Genay, France). Olive leaves used for preparing olive leaf extract (OLE) and highly purified oleuropein (99.3) were obtained from olive trees grown in Sfax, Tunisia. Acetonitrile, acetic acid, and phosphoric acid of high performance liquid chromatography (HPLC) grade were also purchased from Wako Pure Chemical Industries, Ltd. (Osaka, Japan). 


\subsection{Preparation of OLE and highly purified oleuropein}

138

139

140

141

142

\subsubsection{Preparation and phenolic characterization of $O L E$}

We used aqueous OLE because its simplicity, no use of chemical and toxic organic solvents, is of great interest for food and cosmetic applications. It was prepared according to the procedure described by (Bouaziz \& Sayadi, 2005) with slight modifications. Briefly, fresh olive leaves of the Chemlali cultivar of Oleaeuropaea L., harvested in April 2012, were dried in a microwave oven at $2500 \mathrm{~W}$ for $5 \mathrm{~min}$, and subsequent milling (Moulinex228 Coffee Grinder, Paris, France) yielded a fine olive leaf powder. Then, $200 \mathrm{~g}$ of the powder was added to $2 \mathrm{~L}$ of deionized water. The mixture was then shaken overnight in dark at $30{ }^{\circ} \mathrm{C}$ using a laboratory scale shaker (Lab Companion SI-600, GMI Inc., Ramsey, USA). Next, the resultant aqueous solution was centrifuged at $3000 \mathrm{rpm}$ for $20 \mathrm{~min}$ using a centrifuge (Hettich Rotina 420R, DJB Labcare, Buckinghamshire, UK) and then filtered using a Whatman glass microfiber filter (GF/F, Sigma-Aldrich Co. LLC, St. Louis, USA). The filtered solution was concentrated in a rotary evaporator (Eyela DTC-21, Tokyo Rikiakikai Co., Ltd., Tokyo, Japan) at $40{ }^{\circ} \mathrm{C}$, and the residue obtained was freeze-dried overnight using a freeze dryer (Eyela FD-81, Tokyo Rikakikai Co., Tokyo, Japan). The freeze-dried residue was stored in dark at $-20{ }^{\circ} \mathrm{C}$ prior to analysis.

The phenolic profile of the OLE prepared here was obtained using an Agilent series 1260 HPLC-DAD instrument (Agilent Technologies, Waldbronn, Germany). The OLE was dissolved in water that was purified through a hydrophilic membrane cellulose acetate filter (Millipore Co., Billerica, USA) with a pore size of $0.2 \mu \mathrm{m}$. Chromatographic separations were carried out on a Zorbax Eclipse XDB-C18 column, serial number USNH027266 (4.6 mm I.D. $\times 250 \mathrm{~mm} \times 3.5 \mu \mathrm{m}$ particle size), maintained at $40{ }^{\circ} \mathrm{C}$. The mobile phase was $0.1 \%$ acetic acid in water (A) versus $100 \%$ acetonitrile (B), and the total running time was $50 \mathrm{~min}$. The 
161

162

163

164

165

elution conditions applied for phenolic compounds were: 0-22 min, 10-50\% B; 22-32 min, 50-100\% B; 32-40 min, $100 \% \mathrm{~B} ; 40-44 \mathrm{~min}, 100-10 \% \mathrm{~B}$. The column was washed and reconditioned after each analysis using $100 \%$ acetonitrile. The flow rate of the mobile phase was $0.5 \mathrm{~mL} / \mathrm{min}$, and the injection volume of the OLE samples was $10 \mu \mathrm{L}$. Detection was performed using a diode array detector (DAD), and the chromatograms were recorded at $\lambda=$ $254 \mathrm{~nm}$ for oleuropein, at $280 \mathrm{~nm}$ for tyrosol, and at $330 \mathrm{~nm}$ for flavonoids (luteolin, apigenin-7-O glucoside, and verbascoside). Quantification was performed by external calibration with standards.

\subsubsection{Purification of oleuropein using semipreparative HPLC}

Oleuropein was purified from the OLE using semipreparative HPLC (Knauer, Germany). The experiment was conducted on a model liquid chromatograph fitted with a eurospher C18 column (Knauer, Germany) with dimensions of $25 \mathrm{~cm} \times 8 \mathrm{~mm}$ I.D. Here, $0.1 \%$ phosphoric acid aqueous solution (A) and 100\% acetonitrile (B) were used as the mobile phases at a flow rate of $5 \mathrm{~mL} / \mathrm{min}$. The gradient was kept at $20 \% \mathrm{~B}$ for $3 \mathrm{~min}$, increased to $100 \% \mathrm{~B}$ at $33 \mathrm{~min}$, kept there until $38 \mathrm{~min}$, and finally decreased to $20 \% \mathrm{~B}$ at $40 \mathrm{~min}$. An eluted peak of oleuropein was collected using a model sampler. The collected oleuropein solution was pooled, and then its solvent was removed by freeze-drying. The purity level of oleuropein was determined using LC-MS/MS (Agilent 1100 LC, Germany) equipment following the method of (Bouaziz, Feki, Ayadi, Jemai, \& Sayadi, 2010).

\subsection{Interfacial tension and viscosity measurements}

Interfacial tension measurements were carried out using aqueous solutions of OLE or highly purified oleuropein. Initially, OLE (0-50 wt \% (g/100g)) and highly purified oleuropein (0-0.8 $\mathrm{wt} \%$ ) were separately dissolved in Milli-Q water in concentrations ranges $0-50 \mathrm{wt} \%$ and 0 
$0.8 \mathrm{wt} \%$, respectively. The aqueous phase samples were prepared by successive dilution of the initial aqueous solution with the highest concentration. The most concentrated samples were serially diluted, resulting in aliquots of different concentrations, prior to measurement. The freeze-dried OLE and highly purified oleuropein (99.3\% purity) used were brownish crystalline powders. Four triglyceride oils with different carbon-chain lengths and different degrees of purity (extra virgin olive oil, refined olive oil, refined soybean oil, and MCT) were used as the oil phase. Prior to interfacial tension measurements, the densities of the aqueous and oil phases were determined using a digital density meter (DMA 35N, Anton Paar GmBH, Graz, Austria). Interfacial tension was determined using a pendant drop tensiometer analyzer (PD-W, Kyowa Interface Science Co., Ltd., Saitama, Japan); following a drop-down approach (Bucak \& Rende, 2014). Each aqueous solution was loaded in a syringe and ejected to form a drop at a needle tip submerged in an oil solution. The needle tip was positioned on an optical bench between a light source and CCD camera in order to record the drop formed. Interfacial tension was calculated using the Young-Laplace equation of capillarity. Each sample was measured at least 10 times to ensure reproducibility, and the average values were used for data analyses.

The dynamic viscosities of the two liquid phases were also determined using glass capillary viscometers (SO-200 and SO-350, Shibata Scientific Technology Ltd., Tokyo, Japan). All measurements were carried out at $25^{\circ} \mathrm{C}$.

\subsection{Determination of aggregation parameters of oleuropein at oil-water interface}

The adsorption of oleuropein at the oil-water interface could be estimated by the decrease in interfacial tension when adding oleuropein to the aqueous phase. The relationship between the amount of oleuropein adsorbed at the oil-water interface (i.e., surface 


$$
\Gamma=-\frac{1}{R T}\left(\frac{d \gamma}{d \ln C}\right)_{T}
$$

209

210

211

212

excess concentration, $\left.\Gamma\left(\mathrm{mol} / \mathrm{m}^{2}\right)\right)$ and the interfacial tension, $\gamma(\mathrm{N} / \mathrm{m})$, is given by the Gibbs equation (Lucas et al., 2010). Here, $R$ is the gas constant $\left(8.314 \mathrm{~J} \mathrm{~mol}^{-1} \mathrm{~K}^{-1}\right), T$ is the absolute temperature $(\mathrm{K})$, and $C$ is the concentration of oleuropein in the water phase $(\mathrm{mol} / \mathrm{L})$.

Knowing $\Gamma$, the area occupied by one molecule adsorbed at the interface, $\sigma\left(\AA^{2}\right)$, can be estimated from:

$$
\sigma=\frac{10^{16}}{N_{A} \Gamma}
$$

where $N_{A}$ is Avogadro's number $\left(6.022 \times 10^{23} \mathrm{~mol}^{-1}\right)$.

\subsection{Preparation of oleuropein-stabilized $\mathrm{O} / \mathrm{W}$ emulsions using $M C$ emulsification}

\subsubsection{Experiment setup and emulsification procedure}

The laboratory-scale MC emulsification setup used for this study is illustrated in Fig. 2. This setup consists of a custom-made module equipped with a silicon grooved MC array plate (CMS6-2, EP Tech. Co. Ltd., Hitachi, Japan), a liquid chamber to supply the dispersed phase, a syringe pump (Model 11, Harvard Apparatus Inc., MA, USA) to supply the continuous phase, and a microscope video system to monitor and record emulsification behavior in real time. The hydrophilically treated MC array plate was firmly attached to an optically flat glass plate during module assembly. MC emulsification was carried out using a $0.6 \mathrm{wt} \%$ highly purified oleuropein aqueous solution as the continuous phase and triacylglycerol oil as the dispersed phase. Prior to usage, the aqueous solution was filtered using a cellulose acetate 
filter with a pore size of $0.45 \mu \mathrm{m}$. The MC array plate, flow directions of the two phases, and droplet generation through the MCs are depicted in Fig. $2 \mathrm{~b}$. The $25 \mathrm{~mm} \times 28 \mathrm{~mm} \mathrm{MC}$ array plate consists of a total of 1070 parallel MCs $(5-\mu \mathrm{m}$ depth and $8-\mu \mathrm{m}$ width) with terraces $(5-$ $\mu \mathrm{m}$ depth and 40- $\mu \mathrm{m}$ length) outside them. Deeply etched wells (100- $\mu \mathrm{m}$ depth) were also fabricated outside each MC array. Oil droplets were formed by pressurizing the dispersed phase supplied in the module through the MC arrays into the wells initially filled with continuous phase. The hydraulic pressure applied to the dispersed phase $\left(\Delta P_{\mathrm{d}}\right)$, was calculated using:

$$
\Delta P_{\mathrm{d}}=\rho_{\mathrm{d}} g \Delta h_{\mathrm{d}}
$$

where $\rho_{\mathrm{d}}$ is the density of the oil phase $\left(\mathrm{kg} \cdot \mathrm{m}^{-3}\right), g$ is acceleration due to gravity $\left(\mathrm{m} \cdot \mathrm{s}^{-2}\right)$, and $\Delta h_{\mathrm{d}}$ is the difference in the hydraulic heads between the MC array plate and top surface of the oil phase in the reservoir. Real time optical microscopy was used for tuning droplet generation from the MCs during the experiments. All the experiments were performed at room temperature $\left(\sim 25^{\circ} \mathrm{C}\right)$.

\subsubsection{Droplet size measurement}

Micrographs of the resultant droplets were recorded for droplet size measurement. Their size was calculated as the number-weighted average droplet diameter $\left(d_{\mathrm{av}}\right)$, which was determined from the diameters of 100 droplets. The diameter of each droplet was measured using image processing software (WinRoof ver. 5.6, Mitani Co., Fukui, Japan). Droplet size distribution was expressed as the coefficient of variation (CV), defined as 100 times the ratio between the standard deviation of droplet size measurement and $d_{\mathrm{av}}$.

\section{Results and discussion}




\subsection{Compositional characterization of OLE and highly purified oleuropein}

254

255

256

257

HPLC chromatograms of the OLE and highly purified oleuropein are depicted in Fig. 3a and $3 \mathrm{~b}$, respectively. On the basis of an external standard determination, six major phenolic compounds were identified and quantified in the aqueous OLE (Fig. 3a): tyrosol $(0.17 \%)$, luteolin glycoside $(1.1 \%)$, verbascoside $(1.5 \%)$, luteolin $(0.22 \%)$, apigenin-7-O glucoside (1.5\%), and oleuropein (35.4\%). These values are expressed as the weight ratio of each compound to the freeze dried extract. The extraction yield of oleuropein, defined as the weight ratio of oleuropein to the initial dried olive leaves, was around $15 \%$. The resulting oleuropein yield from our aqueous extract was similar to that from methanolic extract (Bouaziz \& Sayadi, 2005) but higher than that from ethanolic extract (Flemmig, Kuchta, Arnhold, \& Rauwald, 2011). The difference in composition and concentration of phenolic compounds among the studied OLEs (Bouaziz \& Sayadi, 2005; Flemmig et al., 2011; Poudyal, Campbell, \& Brown, 2010) could be associated with the polarity of extraction solvents that may affect the diffusion of phenolic compounds into the extraction media. The phenolic composition could also be influenced by differences in the variety, season, and location of collection (Ben Salah \& Abderraba, 2012). The chromatogram of Fig. 3b depicts a sharp monomodal peak of oleuropein at a retention time of $15 \mathrm{~min}$. Oleuropein purity, determined by LC-MS-MS, was $99.3 \%$.

\subsubsection{Interfacial activity of OLE and highly purified oleuropein}

Variation of the interfacial tension at a soybean oil-water interface in the presence of different concentrations of OLE or highly purified oleuropein is presented in Fig. 4. Both solutes exhibited a significant interfacial activity with dose dependence. Although the oleuropein 
concentration is different at the same solute concentration, highly purified oleuropein and OLE showed similar trends in lowering interfacial tension at concentrations below the maximum solubility of oleuropein. We suppose that the presence of other surface active compounds in olive leaves, mainly proteins, induced a further decrease of interfacial tension values for the OLE-containing systems ((Boudhrioua, Bahloul, Ben Slimen, \& Kechaou, 2009). OLE showed a linear decrease in the interfacial tension followed by a plateau in the data, indicating saturation of the soybean oil-water interface by components of OLE at a critical micelle concentration $(\mathrm{CMC})$ of $0.1 \mathrm{wt} \%$. Subsequently, interfacial tension slowly decreased to about $9 \mathrm{mN} / \mathrm{m}$ at an OLE concentration of $50 \mathrm{wt} \%$. However, the interfacial tension for the purified oleuropein-containing system decreased to $18.2 \mathrm{mN} / \mathrm{m}$, but it did not reach the $\mathrm{CMC}$ because of the low maximum oleuropein solubility in water. Ultracentrifugation (40000 $\mathrm{rpm} / 30 \mathrm{~min}$ ) and subsequent HPLC analysis showed that OLE could be solubilized in the tested range of concentrations. Solubility determination of OLE was based on oleuropein concentration in the initial freeze-dried OLE sample. OLE is a mixture of carbohydrates, proteins, and phenolic compounds (Laguerre et al., 2009; Wang, Vignani, Scali, \& Cresti, 2006). Oleuropein has protein binding properties (Bao et al., 2007), which could cause its higher solubility in diluted OLE.

\subsubsection{Aggregation parameters of oleuropein at different triglyceride oil-water interfaces}

The influence of the molecular concentration of highly purified oleuropein on interfacial tension is semilogarithmically presented in Fig. 5. The triglyceride oils used here are refined olive oil, extra virgin olive oil, soybean oil, and MCT oil.

Interfacial tension in the presence of oleuropein depended on the oil type, especially at very low oleuropein concentrations. In the absence of oleuropein, MCT oil had the highest interfacial tension, $33.8 \mathrm{mN} / \mathrm{m}$, followed by refined soybean oil $(31.5 \mathrm{mN} / \mathrm{m})$, refined olive 
oil $(28.4 \mathrm{mN} / \mathrm{m})$, and extra virgin olive oil $(25.3 \mathrm{mN} / \mathrm{m})$. Small amounts of mono- and diglycerides, free fatty acids, and phospholipids may be present, depending on the source and processing conditions. Refined oils are nearly free of phospholipids, whereas extra virgin olive oil usually contains some interfacial active impurities (e.g., phenolic compounds and carotenoids) (Gaonkar, 1989). These impurities are assumed to adsorb at the oil-water interface, leading to a further decrease in interfacial tension.

The Gibbs adsorption isotherm plot, which expresses the variation of interfacial tension as a function of emulsifier concentration, typically consists of a linear decrease in interfacial tension followed by a plateau of data. The slope of the linear portion is crucial for determining the aggregation parameters of an emulsifier (Santanu Paria, 2005). Linear decreases in interfacial tension in the presence of highly purified oleuropein were observed from $10^{-4}$ to $10^{-2} \mathrm{~mol} / \mathrm{L}$, regardless of the oil type (Fig. 5b). Aggregation parameters of oleuropein obtained from the linear fittings in Fig. $5 \mathrm{~b}$ are summarized in Table 1 . The number of oleuropein molecules adsorbed per unit area (Eq. 1) ranged from $5.7 \times 10^{-7}$ to $8.2 \times 10^{-7} \mathrm{~mol} / \mathrm{m}^{2}$. The equations presented in Fig. $5 \mathrm{~b}$ fitted the interfacial tension data well $\left(R^{2}>0.98\right)$. The area occupied by an oleuropein molecule at the oil-water interface (Eq. 2) was 202-289 $\AA^{2}$. The smallest areas per molecule were obtained for MCT oil, and the largest were obtained for extra virgin olive oil. The difference may be ascribed to the smaller size of the MCT oil molecule compared to long-chain triglyceride oils molecules. The interaction of the hydrocarbon chain of MCT and oleuropein will vary, altering its packing behavior at the $\mathrm{O} / \mathrm{W}$ interface (Trotta, Gallarate, Pattarino, \& Carlotti, 1999). The higher quantity of impurities present in the nonrefined oils may compete with oleuropein molecules at the interface, resulting in the larger areas obtained. However, these data exceed the maximal projection area 
322 of oleuropein (138 $\AA$ ) (www.Chemicalize.org), indicating that oleuropein molecules are not

323 closely packed because the maximum oleuropein concentration is lower than the CMC.

324

325

326

327

\subsubsection{Mechanism of interfacial activity of oleuropein}

The interfacial activity of oleuropein in the triglyceride oil-water systems is assumed to be due to the presence of hydrophilic and hydrophobic parts in the oleuropein molecule. The hydrophilic head group would be formed by the glucose moiety, whereas the hydrophobic tail would consist of the oleuropein aglycone moiety. Our assumption is further based on the presence of ester bonds in the oleuropein molecule that would approach its activity to those of glucose esters surfactants (Holmberg 2001). The large areas occupied by oleuropein molecules at the triglyceride-water interface are considered to reflect a low-ordered packing, which is mainly due to the structure of the hydrophobic moiety. Because the occupied area per a surfactant molecule is independent of its head group size (Ruiz 2008), the short and ramified hydrophobic chain in the oleuropein molecule might cause a high $\mathrm{CMC}$ value. Chain branching in hydrocarbon surfactants gives a higher $\mathrm{CMC}$, and an increase in the carbon number gives a lower $\mathrm{CMC}$. The benzoic ring is equivalent to a carbon chain of $\sim 3.5$ (Ruiz 2008), then its presence in the hydrophobic moiety would improve the interfacial activity of oleuropein. Oleuropein has a higher interfacial activity than other olive-derived antioxidants (Di Mattia et al., 2010). Considering that oleuropein molecules are hydrophilic with a partition coefficient of $6 \times 10^{-4}$ (octanol/water) (Rodis, Karathanos, \& Mantzavinou, 2002), we will discuss in a later section the emulsifying ability of oleuropein for preparing $\mathrm{O} / \mathrm{W}$ emulsions using MC emulsification.

\subsection{Preparation of oleuropein-stabilized $\mathrm{O} / W$ emulsions using $M C$ emulsification}

\subsubsection{Oil droplet generation in the absence of cross-flowing continuous phase}


345 Typical examples of the generation of triglyceride oils using $\mathrm{MC}$ emulsification in the 346 absence of a cross-flowing continuous phase are depicted in Fig. 6a. The value of $\Delta P_{\mathrm{d}}$ was 347 just above the breakthrough pressure, which is the minimum pressure required for generating 348 droplets. The $\Delta P_{\mathrm{d}}$ value, which ranged between 6 and $9 \mathrm{kPa}$, depended on the oil type (i.e., interfacial tension). For extra virgin olive oil-, refined olive oil-, and soybean oil-containing systems, the oil phase that passed through the MCs almost inflated on the terrace with a symmetrical shape, and uniformly sized oil droplets were periodically generated from spontaneous transformation of the oil-water interface over the MC outlets (Fig. 6a, left). Coalescence of the generated oil droplets was not observed in the well. For extra virgin olive oil, $d_{\mathrm{av}}$ was $25.1 \mu \mathrm{m}$ and CV was $4.9 \%$; for refined olive oil, $d_{\mathrm{av}}$ was $25.2 \mu \mathrm{m}$ and CV was 4.7\%; and for refined soybean oil, $d_{\text {av }}$ was $27.2 \mu \mathrm{m}$ and $\mathrm{CV}$ was $5.2 \%$, demonstrating the monodispersity of these $\mathrm{O} / \mathrm{W}$ emulsions. The refining of olive oil did not affect the preceding droplet size and droplet size distribution data, suggesting that the presence of extra minor compounds in extra virgin olive oil did not negatively affect droplet generation. Droplet generation for the MCT oil-containing system appeared to be similar to the other systems. However, the resulting uniformly sized droplets were unstable against coalescence, and some droplets stuck on the well surface (Fig. 6a, right). Coalescence of the MCT oil droplets resulted in greater $d_{\mathrm{av}}(31.5 \mu \mathrm{m})$ and $\mathrm{CV}(22.9 \%)$, and the prepared $\mathrm{O} / \mathrm{W}$ emulsion was polydisperse. The MCT oil is composed by the simple combination of caprylic acid (C8:0) and capric acid (C10:0) fatty acids resulting in its lower viscosity (Table 2), which causes larger droplet sizes and slight solubility in water. Consequently, coalescence of MCT oil droplets and their adhesion on the well surface can be attributed mainly to a hydrophobicity 367 below that of olive and soybean oils, whose major components are long-chain 368 triacylglycerides. In our previous MC emulsification study, these unstable behaviors were not 
observed when using sodium dodecyl sulfate as an emulsifier (G. Vladisavljević, Kobayashi, \& Nakajima, 2011), indicating that oleuropein has weaker interfacial activity.

\subsubsection{Effect of flow rate of continuous phase on oil droplet generation}

The influence of the flow rate of continuous phase $\left(Q_{\mathrm{c}}\right)$ on the $d_{\mathrm{av}}$ and CV of the oleuropeinstabilized $\mathrm{O} / \mathrm{W}$ emulsions is presented in Fig. 7. When using extra virgin olive oil-, refined olive oil-, and soybean oil-containing systems, the $d_{\mathrm{av}}$ and CV values were hardly affected by

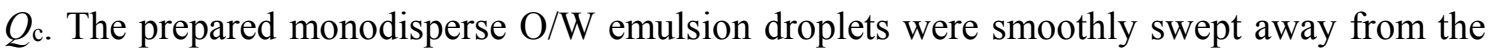
terrace outlet without coalescence and sticky aggregation (Fig. 6b, left). The flow state of the continuous phase flowing in the well can be evaluated using a dimensionless Reynolds number, defined as:

$$
\operatorname{Re}_{c}=\frac{\rho_{c} U_{c} d_{\text {eq,well }}}{\eta_{c}}=\frac{\rho_{c} U_{c}\left(A_{\text {well }} / L_{\text {well }}\right)}{\eta_{c}},
$$

where $R e_{\mathrm{c}}$ is the Reynolds number of the cross-flowing continuous phase, $\rho_{\mathrm{c}}$ is the density of the continuous phase, $U_{\mathrm{c}}$ is the velocity of the continuous phase, $d_{\text {eq,well }}$ is the equivalent cross-sectional diameter of the well, $\eta_{\mathrm{c}}$ is the viscosity of the continuous phase, $A_{\text {well }}$ is the cross-sectional area of the well, and $L_{\text {well }}$ is the wetted perimeter of the well. The maximum $R e_{\mathrm{c}}$ in this study was 2.3 , suggesting laminar flow of the cross-flowing continuous phase. The cross-flowing continuous phase has a parabolic velocity distribution in the well, and the velocity of the continuous phase around the dispersed phase expanding outside the terrace is much slower than the $U_{\mathrm{c}}$ value in Eq. 5 (Isao Kobayashi, Wada, Uemura, \& Nakajima, 2010). This reduced velocity of the continuous phase leads to a reduction of the drag force acting on the expanding droplets, which would be the reason for the almost constant $d_{\mathrm{av}}$ and CV values in the flow rate ranges of the continuous phase applied in this study. 
However, the $d_{\mathrm{av}}$ and $\mathrm{CV}$ values of the MCT oil droplets stabilized by oleuropein decreased with increasing $Q_{\mathrm{c}}$ from 0 to $4 \mathrm{~mL} / \mathrm{h}$, reaching $d_{\mathrm{av}}$ and $\mathrm{CV}$ values similar to those obtained for the other types of oils. Their $d_{\mathrm{av}}$ and CV became almost constant at a $Q_{\mathrm{c}}$ of $4-8 \mathrm{~mL} / \mathrm{h}$. The

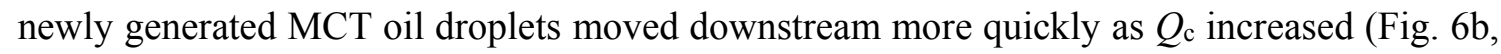
right), possibly preventing contact between the droplet and tip of the dispersed phase that began to expand from the terrace outlet.

\subsubsection{Effect of continuous MC emulsification on oil droplet generation}

Continuous MC emulsification using four different types of oils was performed for $15 \mathrm{~h}$ at a $Q_{\mathrm{c}}$ of $4 \mathrm{~mL} / \mathrm{h}$. Typical droplet generation behaviors of $\mathrm{O} / \mathrm{W}$ emulsion droplets after $15 \mathrm{~h}$ of MC emulsification are depicted in Fig. 6c. Uniformly sized droplets of extra virgin olive oil, refined olive oil, and soybean oil were successfully generated during the entire operation period. A micrograph of droplet generation behavior when using refined soybean oil is listed in Fig. 6c (left). For extra virgin olive oil, $d_{\mathrm{av}}$ was $25.3 \mu \mathrm{m}$ and $\mathrm{CV}$ was $5.1 \%$; for refined olive oil, $d_{\mathrm{av}}$ was $25.6 \mu \mathrm{m}$ and CV was $4.9 \%$; and for refined soybean oil, $d_{\mathrm{av}}$ was $27.4 \mu \mathrm{m}$ and $\mathrm{CV}$ was $5.3 \%$. The oil droplets generated in this case barely stuck to the well surface and did not coalesce. Moreover, these oil droplets did not coalesce for $15 \mathrm{~h}$ after collection (data not shown), suggesting that an oleuropein layer formed on the droplet interface has the ability to prevent droplet coalescence if oils are sufficiently hydrophobic. In contrast, some MCT oil droplets stuck in the well and coalesced with newly generated droplets, resulting in the formation of remarkably large oil droplets near the terrace outlet (Fig. 6c, right). As a result, it was difficult to prepare monodisperse $\mathrm{O} / \mathrm{W}$ emulsions continuously using MCT oil, even in the presence of a cross-flowing continuous phase. From the above observations, it can be concluded that the lower efficiency of oleuropein in producing stable oil droplets when MCT is used might be due to insufficient hydrophobic interactions of the oleuropein aglycone 
416 moiety with MCT; this results in an unstable and insufficient protective film against

417 coalescence. The emulsifying ability of oleuropein could be improved by the modification of 418 the hydrophobic moiety through enzymatic or chemical processes.

\section{Conclusions}

421 Interfacial tension data demonstrated that oleuropein, the major phenolic compound in OLE, 422 is a highly interfacial-active compound. The interfacial area occupied by one oleuropein 423 molecule was quantitatively determined from the Gibbs adsorption theory, suggesting that the 424 oleuropein molecules did not fully cover the triglyceride oil-water interfaces owing to their 425 short hydrophobic moiety. MC emulsification results demonstrated the preparation of 426 monodisperse, oleuropein-stabilized $\mathrm{O} / \mathrm{W}$ emulsions with a $\mathrm{CV}$ of $<5 \%$ when using 427 triglyceride oils of higher hydrophobicity, even in the absence of a cross-flowing continuous 428 phase. MC emulsification using oleuropein as the emulsifier is also capable of continuously 429 preparing monodisperse $\mathrm{O} / \mathrm{W}$ emulsions for $15 \mathrm{~h}$, if appropriate triglyceride oils are used. Our 430 findings are believed to provide new insights for novel food and pharmaceutical applications 431 of oleuropein as a natural emulsifier.

434 The authors are grateful to the Science and Technology Research Partnership for Sustainable Development (SATREPS) Project, financially supported by JICA and JST, Japan. 


\section{Figure captions}

438

439 Fig. 1. Chemical structure of oleuropein.

440

441

Fig. 2. (a) Simplified schematic view of the MC emulsification setup used in this study. (b)

442

443

Schematic top views of a silicon grooved MC array plate CMS6-2 (left) and schematic representation of droplet generation via MCs (right).

444

445

446

Fig. 3. Semipreparative HPLC chromatograms of (a) olive leaf extract, and (b) highly purified oleuropein. Oleuropein, $\lambda=254 \mathrm{~nm}$; tyrosol, $280 \mathrm{~nm}$; and flavonoids, $330 \mathrm{~nm}$.

447

Fig. 4. Variation of interfacial tension between refined soybean oil and water as a function of the concentrations of OLE and highly purified oleuropein.

450

451

Fig. 5. (a) Variation of interfacial tension between the oil and water phases as a function of

452 the concentration of highly purified oleuropein $(\mathrm{mol} / \mathrm{L})$. The data plotted in the dashed

453 rectangle are fitted with equations presented in (b).

454

455

456

457

458

459

Fig. 6. Optical micrographs of oil droplet generation via MCs in the continuous phase containing $0.6 \mathrm{wt} \%$ of oleuropein. (a) Generation of oil droplets in the absence of a crossflowing continuous phase. (b) Generation of oil droplets in the presence of a cross-flowing continuous phase $(4 \mathrm{~mL} / \mathrm{h})$ at the beginning of emulsification. (c) Generation of oil droplets in the presence of a cross-flowing continuous phase $(4 \mathrm{~mL} / \mathrm{h})$ after continuous $\mathrm{MC}$ 460 emulsification for $15 \mathrm{~h}$.

461 
462 Fig. 7. Effect of the continuous phase flow rate on the average droplet diameter $\left(d_{\text {av }}\right)$ and 463 coefficient of variation $(\mathrm{CV})$ of oleuropein-stabilized $\mathrm{O} / \mathrm{W}$ emulsions. Filled symbols 464 represent $\mathrm{d}_{\mathrm{av}}$; open symbols represent $\mathrm{CV}$.

465

466

467

468

469

470

471

472

473

474

475

476

477

478

479

480

481

482

483

484

485

486 


\section{References}

Andreadou, I., Iliodromitis, E. K., Mikros, E., Constantinou, M., Agalias, A., Magiatis, P., Skaltsounis, A. L., Kamber, E., Tsantili-Kakoulidou, A., \& Kremastinos, D. T. (2006). The olive constituent oleuropein exhibits anti-Ischemic, antioxidative, and hypolipidemic effects in anesthetized rabbits. The Journal of Nutrition, 136 (8), 22132219.

Bao, J., Zhang, D. W., Zhang, J. Z., Huang, P. L., Huang, P. L., \& Lee-Huang, S. (2007). Computational study of bindings of olive leaf extract (OLE) to HIV-1 fusion protein gp41. FEBS Lett, 581 (14), 2737-2742.

Bhupinder, B. S. (2013). Surfactants: Pharmaceutical and Medicinal Aspects Journal of Pharmaceutical Technology, Research and Management, 1,11-36.

Bucak, S., \& Rende, D. (2014). Colloid and surface chemistry: A laboratory guide for exploration of the Nano World, pp. 246

Benavente-García, O., Castillo, J., Lorente, J., Ortuño, A., \& Del Rio, J. A. (2000). Antioxidant activity of phenolics extracted from Olea europaea L. leaves. Food Chemistry, 68 (4), 457-462.

Ben Salah, M., \& Manef Abderraba, H. A. (2012). Study of phenolic composition and biological activities assessment of olive leaves from different varieties grown in Tunisia. Medicinal chemistry, 2 (5), 107-111.

Bisignano, G., Tomaino, A., Cascio, R. L., Crisafi, G., Uccella, N., \& Saija, A. (1999). On the in-vitro antimicrobial activity of oleuropein and hydroxytyrosol. Journal of Pharmacy and Pharmacology, 51 (8), 971-974.

Bouaziz, M., Feki, I., Ayadi, M., Jemai, H., \& Sayadi, S. (2010). Stability of refined olive oil and olive-pomace oil added by phenolic compounds from olive leaves. European Journal of Lipid Science and Technology, 112 (8), 894-905. 
Bouaziz, M., \& Sayadi, S. (2005). Isolation and evaluation of antioxidants from leaves of a Tunisian cultivar olive tree. European Journal of Lipid Science and Technology, 107 (7-8), 497-504.

Boudhrioua, N., Bahloul, N., Ben Slimen, I., \& Kechaou, N. (2009). Comparison on the total phenol contents and the color of fresh and infrared dried olive leaves. Industrial Crops and Products, 29 (2-3), 412-419.

Casas-Sanchez, J., Alsina, M. A., Herrlein, M., \& Mestres, C. (2007). Interaction between the antibacterial compound, oleuropein, and model membranes. Colloid and Polymer Science, 285 (12), 1351-1360.

Chaiyasit, W., McClements, D. J., \& Decker, E. A. (2005). The relationship between the physicochemical properties of antioxidants and their Ability to inhibit lipid oxidation in bulk oil and oil-in-water Emulsions. Journal of Agricultural and Food Chemistry, 53 (12), 4982-4988.

Di Mattia, C. D., Sacchetti, G., Mastrocola, D., Sarker, D. K., \& Pittia, P. (2010). Surface properties of phenolic compounds and their influence on the dispersion degree and oxidative stability of olive oil O/W emulsions. Food Hydrocolloids, 24 (6-7), 652658.

Flemmig, J., Kuchta, K., Arnhold, J., \& Rauwald, H. W. (2011). Olea europaea leaf (Ph.Eur.) extract as well as several of its isolated phenolics inhibit the gout-related enzyme xanthine oxidase. Phytomedicine, 18 (7), 561-566.

Gaonkar, A. (1989). Interfacial tensions of vegetable oil/water systems: Effect of oil purification. Journal of the American Oil Chemists' Society, 66 (8), 1090-1092.

Gijsbertsen-Abrahamse, A. J., van der Padt, A., \& Boom, R. M. (2004). Status of cross-flow membrane emulsification and outlook for industrial application. Journal of Membrane Science, 230 (1-2), 149-159. 
537 Goy, R. C., Britto, D. d., \& Assis, O. B. G. (2009). A review of the antimicrobial activity of $538 \quad$ chitosan. Polímeros, 19, 241-247.

539 Han, J., Talorete, T. N., Yamada, P., \& Isoda, H. (2009). Anti-proliferative and apoptotic 540 effects of oleuropein and hydroxytyrosol on human breast cancer MCF-7 cells. $541 \quad$ Cytotechnology, $59(1), 45-53$.

542 Han, J., \& Washington, C. (2005). Partition of antimicrobial additives in an intravenous 543 emulsion and their effect on emulsion physical stability. International Journal of $544 \quad$ Pharmaceutics, 288 (2), 263-271.

545 Jemai, H., El Feki, A., \& Sayadi, S. (2009). Antidiabetic and antioxidant effects of 546 hydroxytyrosol and oleuropein from olive leaves in alloxan-diabetic rats. J Agric Food $547 \quad$ Chem, 57 (19), 8798-8804.

548 Juven, B., Henis, Y., \& Jacoby, B. (1972). Studies on the mechanism of the antimicrobial $549 \quad$ action of oleuropein*. Journal of Applied Bacteriology, 35 (4), 559-567.

550 Kargar, M., Spyropoulos, F., \& Norton, I. T. (2011). The effect of interfacial microstructure 551 on the lipid oxidation stability of oil-in-water emulsions. Journal of Colloid and Interface Science, 357 (2), 527-533.

Kawakatsu, T., Kikuchi, Y., \& Nakajima, M. (1997). Regular-sized cell creation in microchannel emulsification by visual microprocessing method. Journal of the American Oil Chemists' Society, 74 (3), 317-321.

Kobayashi, I., Takano, T., Maeda, R., Wada, Y., Uemura, K., \& Nakajima, M. (2008). Straight-through microchannel devices for generating monodisperse emulsion droplets several microns in size. Microfluidics and Nanofluidics, 4 (3), 167-177.

Kobayashi, I., Wada, Y., Hori, Y., Neves, M. A., Uemura, K., \& Nakajima, M. (2012). Microchannel Emulsification Using Stainless-Steel Chips: Oil Droplet Generation Characteristics. Chemical Engineering \& Technology, 35 (10), 1865-1871. 
Kobayashi, I., Wada, Y., Uemura, K., \& Nakajima, M. (2010). Microchannel emulsification for mass production of uniform fine droplets: integration of microchannel arrays on a chip. Microfluidics and Nanofluidics, 8 (2), 255-262.

Laguerre, M. 1., López Giraldo, L. J., Lecomte, J. r. m., Figueroa-Espinoza, M.-C., Baréa, B., Weiss, J., Decker, E. A., \& Villeneuve, P. (2009). Chain length affects antioxidant properties of chlorogenate esters in emulsion: The cutoff theory behind the polar paradox. Journal of Agricultural and Food Chemistry, 57 (23), 11335-11342.

Lucas, R., Comelles, F., Alcántara, D., Maldonado, O. S., Curcuroze, M., Parra, J. L., \& Morales, J. C. (2010). Surface-Active Properties of Lipophilic Antioxidants Tyrosol and Hydroxytyrosol Fatty Acid Esters: A Potential Explanation for the Nonlinear Hypothesis of the Antioxidant Activity in Oil-in-Water Emulsions. Journal of Agricultural and Food Chemistry, 58 (13), 8021-8026.

Mattia, C., Sacchetti, G., \& Pittia, P. (2011). Interfacial behavior and antioxidant efficiency of olive phenolic compounds in $\mathrm{O} / \mathrm{W}$ olive oil emulsions as affected by surface active agent type. Food Biophysics, 6 (2), 295-302.

McClements., D. (2005). Food emulsions principles, practices, and techniques In (Vol. (second edition), pp. 609).

Medina, I., Lois, S., Alcántara, D., Lucas, R., \& Morales, J. C. (2009). Effect of lipophilization of hydroxytyrosol on its antioxidant activity in fish oils and fish oil-inwater emulsions. Journal of Agricultural and Food Chemistry, 57 (20), 9773-9779.

Omar, S. H. (2010). Oleuropein in Olive and its Pharmacological Effects. Jounal of Scientifica phamaceutica,78, 133-154.

Paiva-Martins, F., Gordon, M. H., \& Gameiro, P. (2003). Activity and location of olive oil phenolic antioxidants in liposomes. Chemistry and Physics of Lipids, 124 (1), 23-36. 
Poudyal, H., Campbell, F., \& Brown, L. (2010). Olive Leaf extract attenuates cardiac, hepatic, and metabolic changes in high carbohydrate-, high fat-fed rats. The Journal of Nutrition, 140 (5), 946-953.

Richards, M. P., Chaiyasit, W., McClements, D. J., \& Decker, E. A. (2002). Ability of surfactant micelles to alter the partitioning of phenolic antioxidants in oil-in-water emulsions. Journal of Agricultural and Food Chemistry, 50 (5), 1254-1259.

Rodis, P. S., Karathanos, V. T., \& Mantzavinou, A. (2002). Partitioning of olive oil antioxidants between oil and water phases. Journal of Agricultural and Food Chemistry, 50 (3), 596-601.

Ruiz, C. C. (2008). Sugar-based surfactants: Fendamentals and Applications. In (Vol. (143), pp. 622).

Santanu Paria, C. M., Kartic C. Khilar. (2005). Adsorption of anionic and non-ionic surfactants on a cellulosic surface. Colloids and Surfaces $A$ : Physicochemical and Engineering Aspects, 252 (2-3), 221-229.

Sitohy, M. Z., Mahgoub, S. A., \& Osman, A. O. (2012). In vitro and in situ antimicrobial action and mechanism of glycinin and its basic subunit. Int J Food Microbiol, 154 (12), 19-29.

Solans, C., Izquierdo, P., Nolla, J., Azemar, N., \& Garcia-Celma, M. J. (2005). Nanoemulsions. Current Opinion in Colloid \& Interface Science, 10 (3-4), 102-110.

Sugiura, S., Nakajima, M., Kumazawa, N., Iwamoto, S., \& Seki, M. (2002). Characterization of spontaneous transformation-based droplet formation during microchannel emulsification. The Journal of Physical Chemistry B, 106 (36), 9405-9409.

Terjung, N., Loffler, M., Gibis, M., Hinrichs, J., \& Weiss, J. (2012). Influence of droplet size on the efficacy of oil-in-water emulsions loaded with phenolic antimicrobials. Food Funct, 3 (3), 290-301. 
611 Trotta, M., Gallarate, M., Pattarino, F., \& Carlotti, M. E. (1999). Investigation of the phase 612 behaviour of systems containing lecithin and 2-acyl lysolecithin derivatives. Int $J$ $613 \quad$ Pharm, $190(1), 83-89$.

614 Viljanen, K., Kylli, P., Hubbermann, E.-M., Schwarz, K., \& Heinonen, M. (2005). 615 Anthocyanin antioxidant activity and partition behavior in whey protein emulsion. $616 \quad$ Journal of Agricultural and Food Chemistry, 53 (6), 2022-2027.

617 Vladisavljević, G., Kobayashi, I., \& Nakajima, M. (2011). Effect of dispersed phase viscosity 618 on maximum droplet generation frequency in microchannel emulsification using 619 620 asymmetric straight-through channels. Microfluidics and Nanofluidics, 10 (6), 1199-

Internet 
Table 1: Aggregation parameters of oleuropein at triglycerides oils-water interfaces

\begin{tabular}{lcc}
\cline { 2 - 3 } & $\begin{array}{c}\text { Surface excess } \\
\text { concentration } \\
\left(\mathbf{m o l} / \mathbf{m}^{\mathbf{2}}\right)\end{array}$ & $\begin{array}{c}\text { Area per molecule } \\
\left(\AA^{\mathbf{2}}\right)\end{array}$ \\
\hline Extra virgin olive oil & $5.74 \times 10^{-7}$ & 289 \\
Refined olive oil & $6.13^{\times} \times 10^{-7}$ & 270 \\
Refined soybean oil & $6.92^{\times} \times 10^{-7}$ & 240 \\
MCT $^{*}$ oil & $8.22 \times 10^{-7}$ & 202 \\
\hline
\end{tabular}

${ }^{*}$ Medium-chain triacylglyceride 
Table 2: Physical properties of triglyceride oils used as the dispersed phase for MC emulsification.

\begin{tabular}{lccc}
\cline { 2 - 4 } & $\begin{array}{c}\text { Viscosity } \\
(\mathbf{m P a} . \mathbf{s})\end{array}$ & $\begin{array}{c}\text { Density } \\
\left(\mathbf{k g} / \mathbf{m}^{\mathbf{3}}\right)\end{array}$ & $\begin{array}{c}\text { Interfacial tension } \\
(\mathbf{m N} / \mathbf{m})\end{array}$ \\
\hline Extra virgin olive oil & 65.3 & 912 & 14.9 \\
Refined olive oil & 62.7 & 911 & 17.7 \\
Refined soybean oil & 49.9 & 914 & 17.3 \\
MCT $^{*}$ oil & 22.9 & 946 & 18.1 \\
\hline
\end{tabular}

*Medium-chain triacylglyceride 


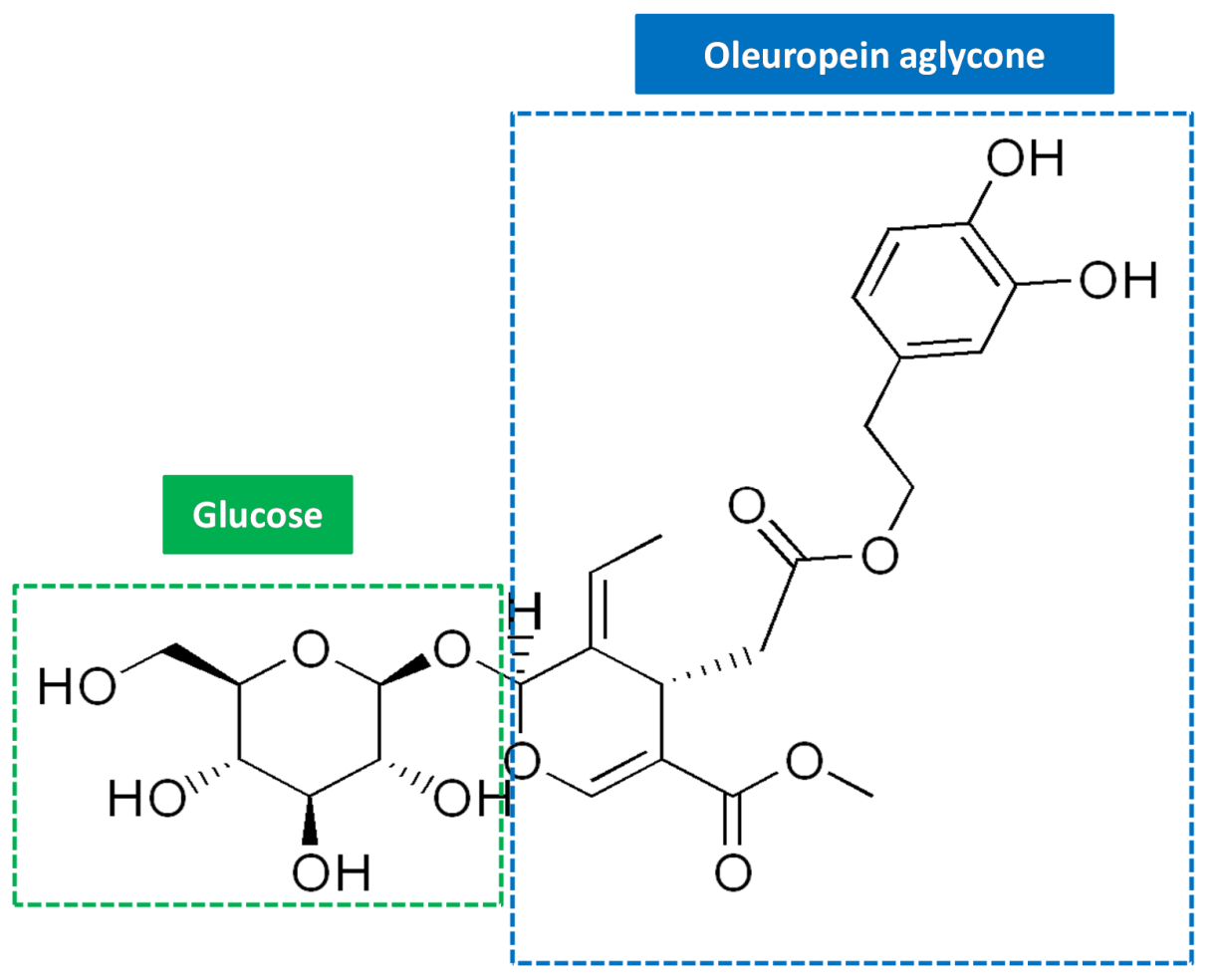

Fig. 1

Souilem et al. 


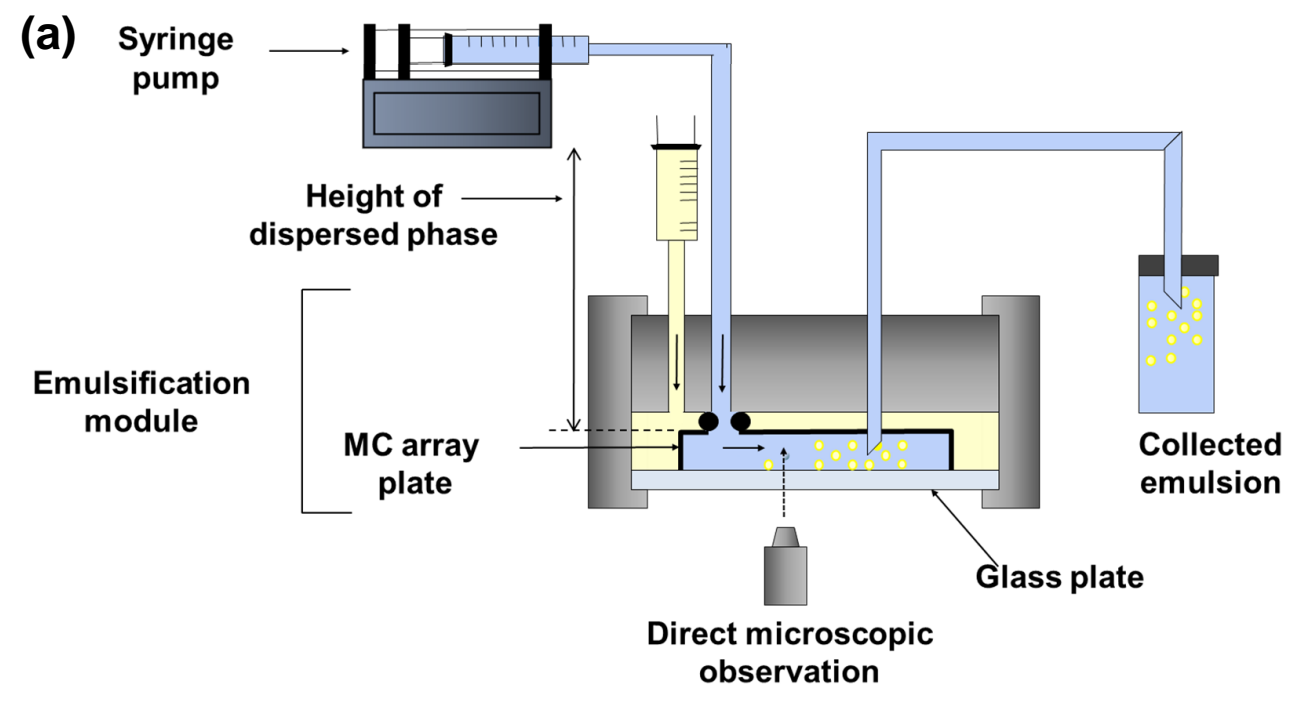

(b)



Fig. 2

Souilem et al. 


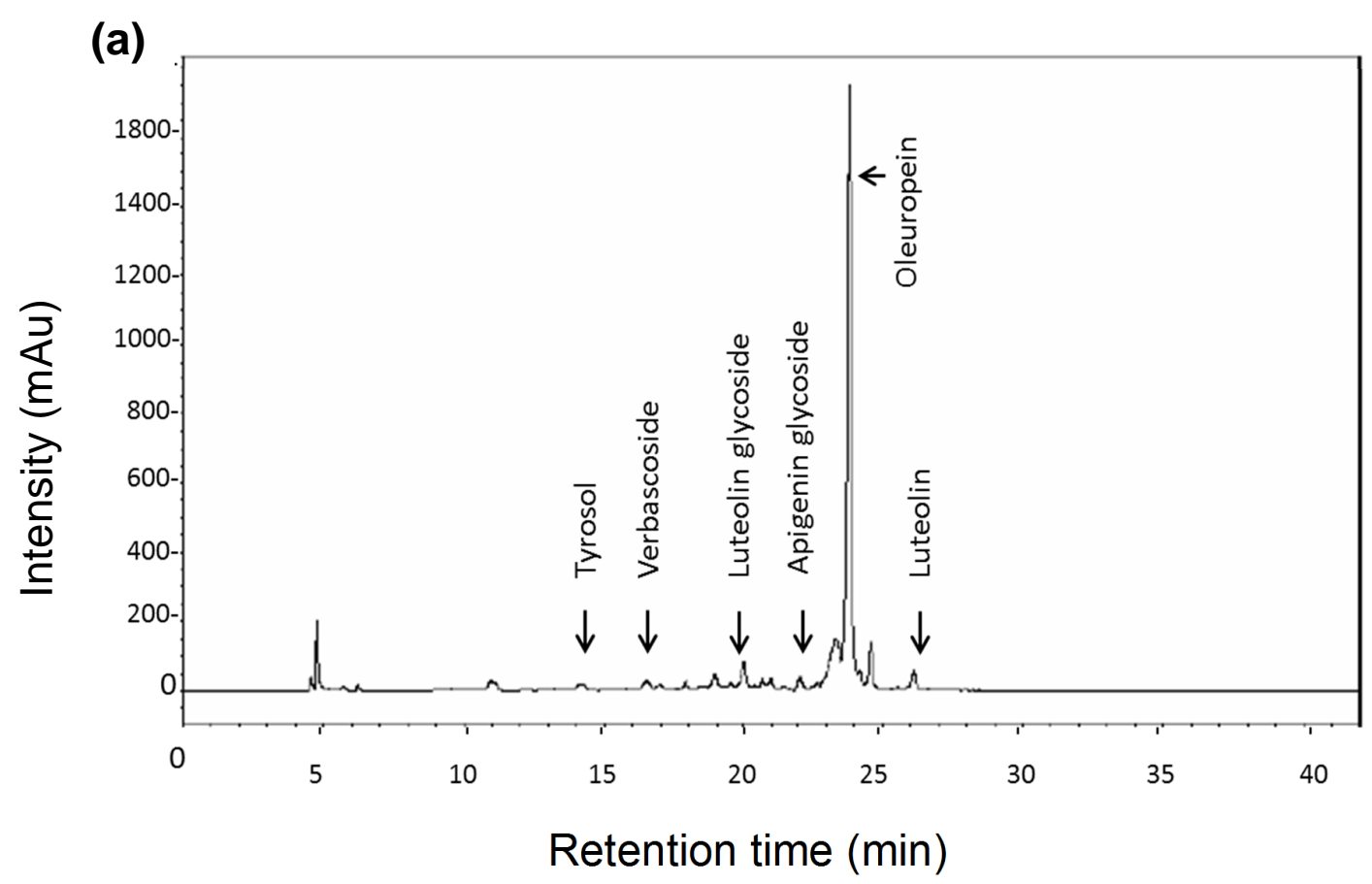

(b)

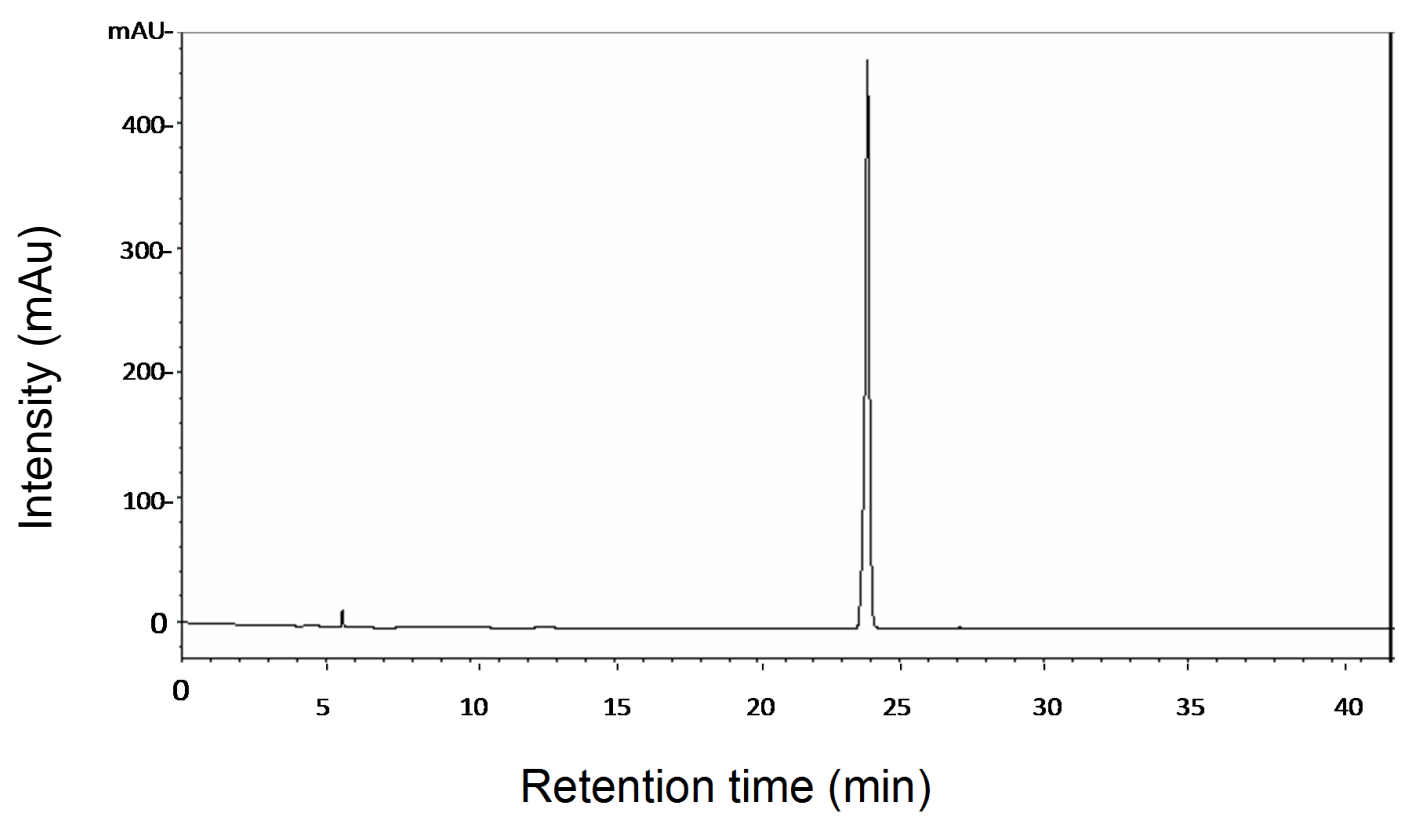

Fig. 3

Souilem et al. 


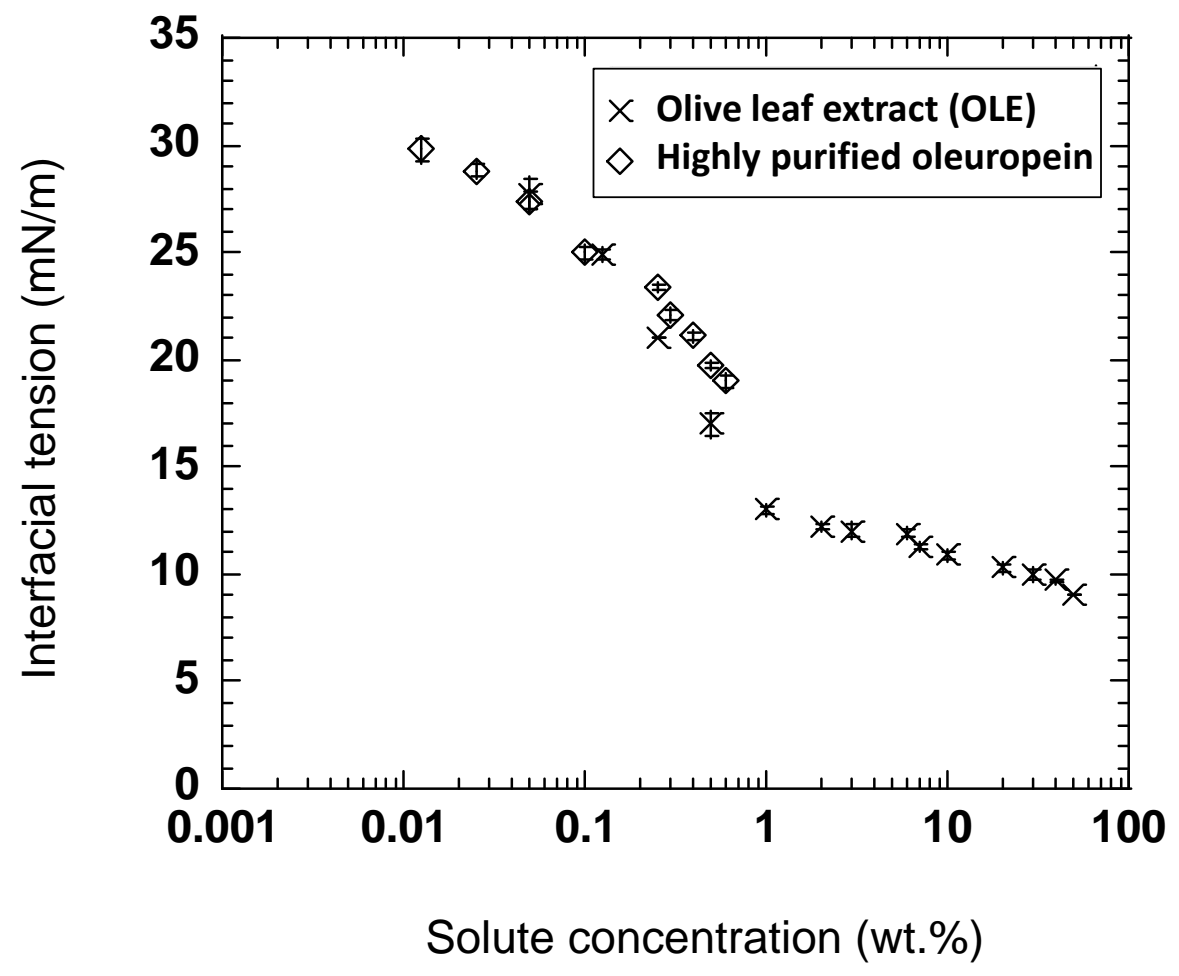

Fig. 4

Souilem et al. 
(a)
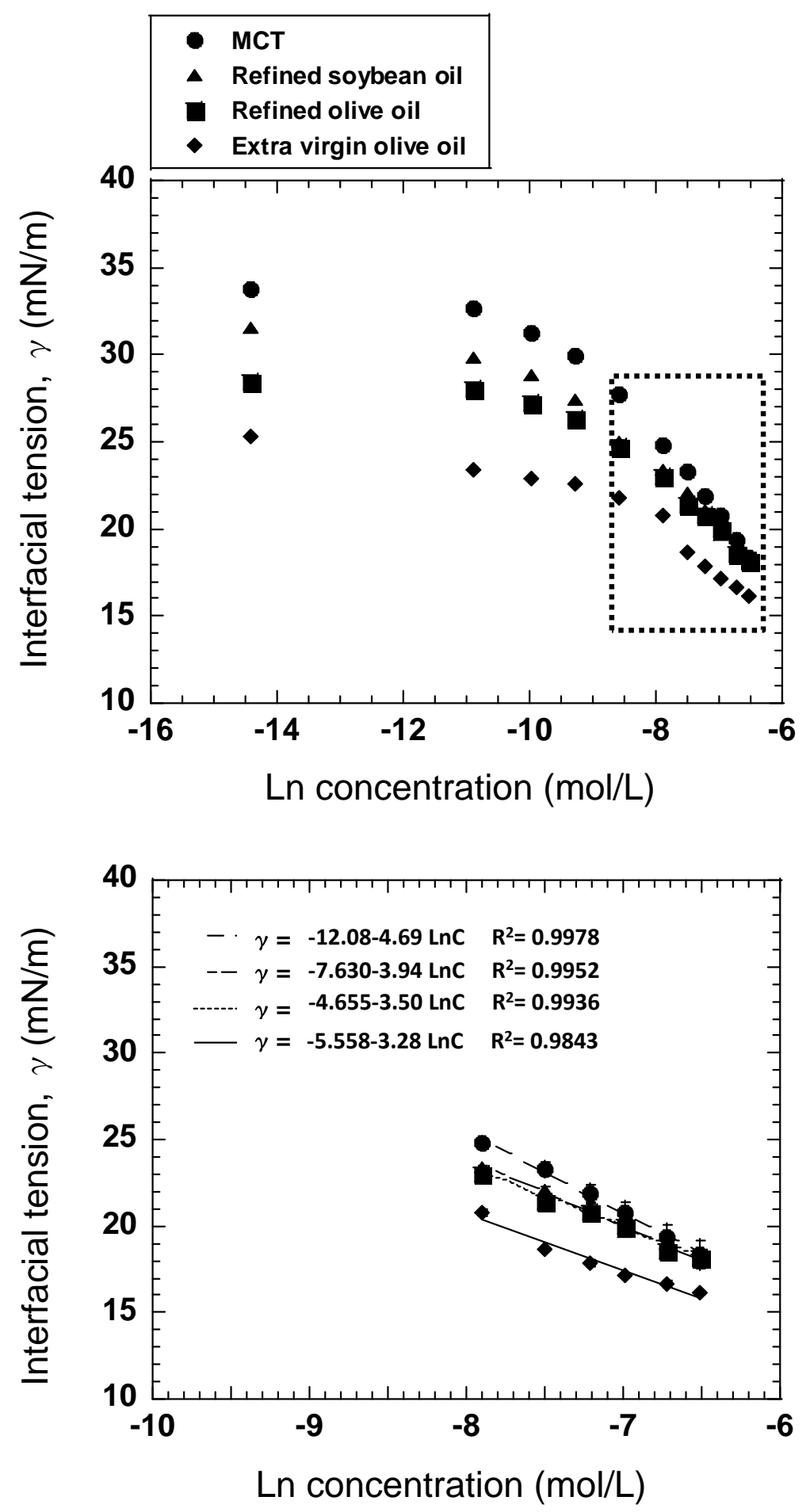

Fig. 5

Souilem et al. 
(a)

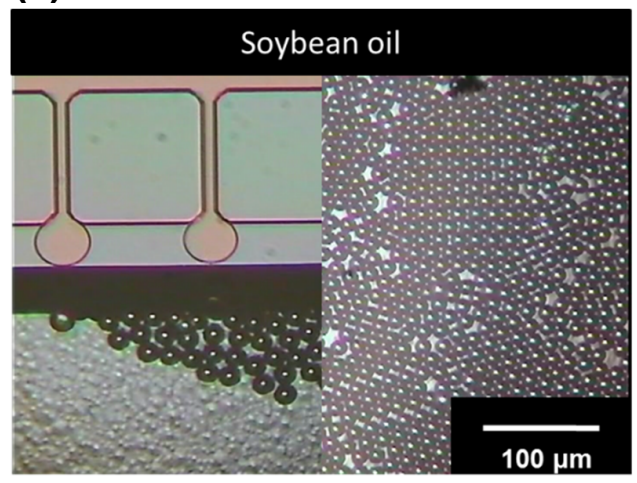

(b)

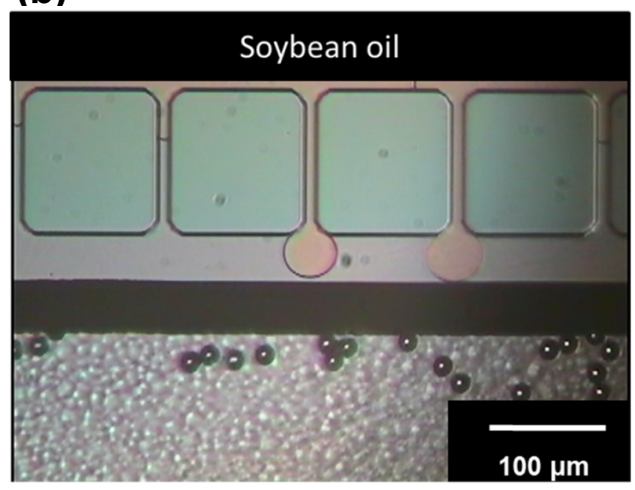

(c)



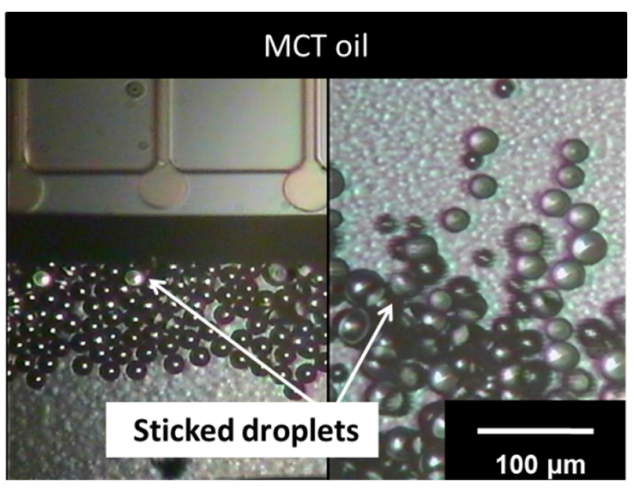

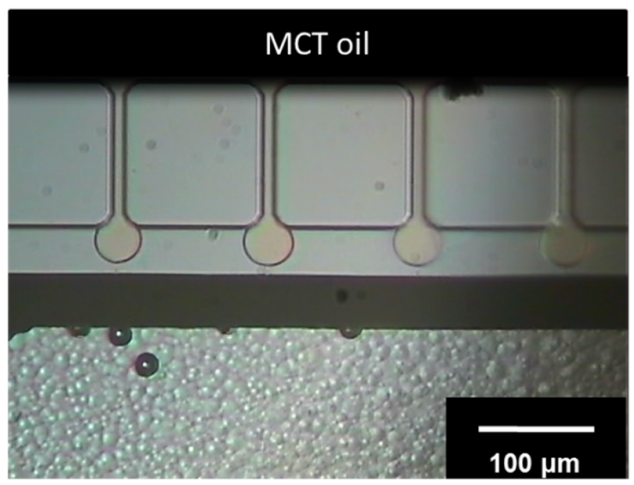

$100 \mu \mathrm{m}$

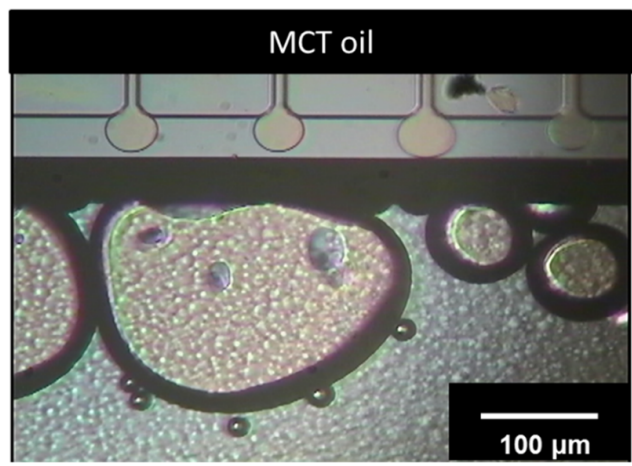

Fig. 6 


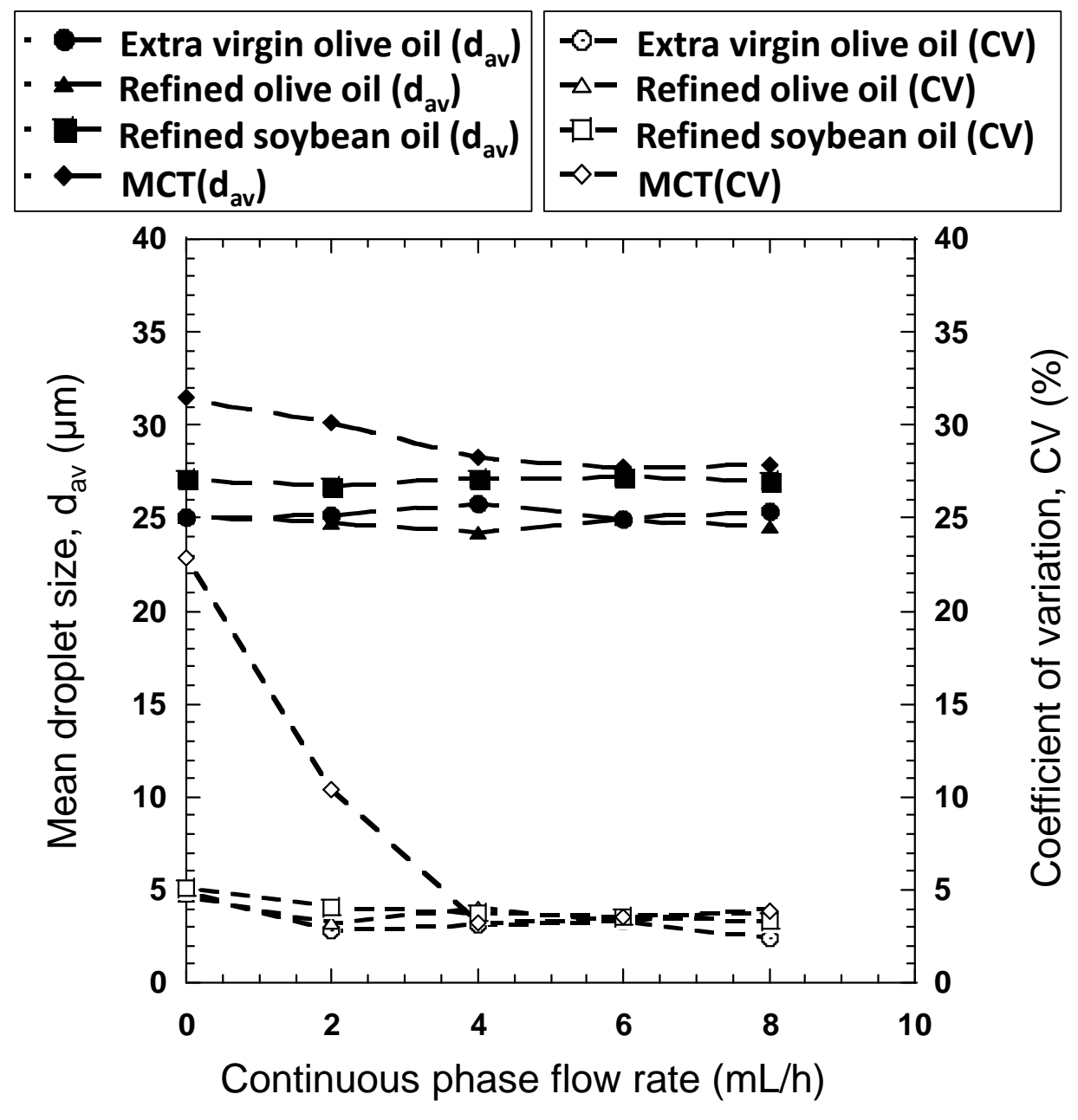

Fig. 7

Souilem et al. 